\title{
Computer-aided assessment of the extra-cellular matrix during pancreatic carcinogenesis: a pilot study
}

\author{
Fabio Grizzi ${ }^{1,2,13^{*}+}$ C , Sirio Fiorino ${ }^{3+}$, Dorina Qehajaj ${ }^{1}$, Adele Fornelli ${ }^{4}$, Carlo Russo ${ }^{5}$, Dario de Biase ${ }^{6}$, \\ Michele Masetti ${ }^{7}$, Laura Mastrangelo ${ }^{7}$, Matteo Zanello ${ }^{7}$, Raffaele Lombardi ${ }^{7}$, Andrea Domanico ${ }^{8}$, \\ Esterita Accogli ${ }^{8}$, Andrea Tura ${ }^{9}$, Leonardo Mirandola ${ }^{11}$, Maurizio Chiriva-Internati ${ }^{11,12}$, Robert S. Bresalier ${ }^{12}$, \\ Elio Jovine ${ }^{7}$, Paolo Leandri ${ }^{3}$ and Luca Di Tommaso ${ }^{2,10}$
}

\begin{abstract}
Background: A hallmark of pancreatic ductal adenocarcinoma is the desmoplastic reaction, but its impact on the tumor behavior remains controversial. Our aim was to introduce a computer -aided method to precisely quantify the amount of pancreatic collagenic extra-cellular matrix, its spatial distribution pattern, and the degradation process.

Methods: A series of normal, inflammatory and neoplastic pancreatic ductal adenocarcinoma formalin-fixed and paraffin-embedded Sirius red stained sections were automatically digitized and analyzed using a computer-aided method.

Results: We found a progressive increase of pancreatic collagenic extra-cellular matrix from normal to the inflammatory and pancreatic ductal adenocarcinoma. The two-dimensional fractal dimension showed a significant difference in the collagenic extra-cellular matrix spatial complexity between normal versus inflammatory and pancreatic ductal adenocarcinoma. A significant difference when comparing the number of cycles necessary to degrade the pancreatic collagenic extra-cellular matrix in normal versus inflammatory and pancreatic ductal adenocarcinoma was also found. The difference between inflammatory and pancreatic ductal adenocarcinoma was also significant. Furthermore, the mean velocity of collagenic extra-cellular matrix degradation was found to be faster in inflammatory and pancreatic ductal adenocarcinoma than in normal.

Conclusion: These findings demonstrate that inflammatory and pancreatic ductal adenocarcinomas are characterized by an increased amount of pancreatic collagenic extra-cellular matrix and by changes in their spatial complexity and degradation. Our study defines new features about the pancreatic collagenic extra-cellular matrix, and represents a basis for further investigations into the clinical behavior of pancreatic ductal adenocarcinoma and the development of therapeutic strategies.
\end{abstract}

Keywords: Pancreatic adenocarcinoma, Extra-cellular matrix, Degradation, Modeling, Fractals

\footnotetext{
${ }^{*}$ Correspondence: fabio.grizzi@humanitasresearch.it

${ }^{\dagger}$ Fabio Grizzi and Sirio Fiorino equally contributed to the manuscript

${ }^{13}$ Histology Core, Humanitas Clinical and Research Center-IRCCS,

Rozzano, Milan, Italy

Full list of author information is available at the end of the article
}

(c) The Author(s) 2019. This article is distributed under the terms of the Creative Commons Attribution 4.0 International License (http://creativecommons.org/licenses/by/4.0/), which permits unrestricted use, distribution, and reproduction in any medium, provided you give appropriate credit to the original author(s) and the source, provide a link to the Creative Commons license, and indicate if changes were made. The Creative Commons Public Domain Dedication waiver (http://creativecommons.org/ publicdomain/zero/1.0/) applies to the data made available in this article, unless otherwise stated. 


\section{Background}

Pancreatic ductal adenocarcinoma (PDAC) represents the seventh leading cause of cancer-related death in the world, with an overall 5 -year survival rate of 5\%. Even localized disease has a 5-year survival rate of only $20 \%-$ $25 \%$ [1]. In contrast to declining trends for other major cancers, death rates are rising in both sexes for PDAC [2]. This malignancy is often diagnosed in an advanced stage, leaving only palliative treatment options [3]. A growing number of studies has considerably improved our knowledge concerning the genetic and epigenetic alterations, the molecular perturbances and the precursor lesions, associated with the onset and the development of this malignancy [4-6]. In particular, changes in telomere length, complex karyotypes and multiple copy number alterations often spanning very large genomic regions, as well as several DNA changes have been reported [3, 7]. The latter consists of activating mutations in oncogenes, such as KRAS, or inactivating alterations in tumor suppressor genes, including P16, TP53, and SMAD4 or in additional genes, such as MLL3, TGFBR2, ARID1A, CDKN2a and ATM [8]. These mutations, observed in non-invasive precursor lesions known as pancreatic intraepithelial neoplasia (PanIN) $[9,10]$, accumulate and drive neoplastic transformation and tumor progression $[9,11,12]$. However, despite these apparently encouraging results, this type of approach has produced no significant impact on the prognosis of PDAC [13]. Therefore, novel pathogenetic models are needed to explain the aggressive biological behavior, and dismal outcome of this malignancy as well as to suggest new and more effective options for its diagnosis and therapy [14]. Accumulating data indicate that not only alterations in malignant epithelial cells, but also extracellular matrix, surrounding cancerous cells play a critical, dynamic and cooperative role in the development of inflammatory as well as cancerous lesions $[15,16]$. Histologically, PDAC is a complex structure, composed of infiltrating neoplastic glands embedded in an intense desmoplastic reaction. The latter consists of an ECM, non-neoplastic activated fibroblasts [17], myofibroblasts, cells of the immune system $[18,19]$, blood and lymphatic vessels. ECM includes collagens, non-collagen glycoproteins, glycosaminoglycans, growth factors and proteoglycans as well as modulators of the cell matrix interaction such as periostin, tenascin C, SPARC (secreted protein acidic and rich in cysteine) and thrombospondin $[20,21]$. This framework represents the bulk of the cancer mass [22]. Interactions between the neoplastic and non-neoplastic cells and ECM have been proposed to stimulate the extensive desmoplastic reaction [23-26]. Although a critical role of stroma in pancreatic carcinogenesis had been recognized for many years, only recently the study of this crucial tissue component has gained a considerable interest and has been considered in clinical practice $[14,27]$.

There is accumulating evidence that while natural stroma can delay or prevent tumorigenesis, abnormal ECM components can promote tumor growth, and that this explains the low therapeutic response of pancreatic cancer patients $[20,28]$. The complex interplay between tumor cells, non-tumoral cells and their ECM products also leads to dynamical changes in the transcriptional program of the cellular components, such as activated fibroblasts, stellate cells and inflammatory cells, which in turn promotes cancer cell motility, resistance to hypoxia and stromal neo-vascularity [29]. To date, mechanisms involved in the initiation and progression of these events are not completely understood. Chronic inflammation exerts a considerable impact in carcinogenesis, by inducing the deposition of a modified ECM tissue with qualitatively and quantitatively altered proteins in comparison with those detectable in normal pancreas. Such a condition is characterized by the progressive development of elevated tensional resistance stresses and high compression forces, both in intracellular and in extracellular compartments, and is associated with the perturbation of homeostasis [30]. This process causes progressive changes in tissue architecture and spatial organization of this organ and induces an increase in its stiffness. Tissue stiffness is now recognized as a risk factor for cancer development not only in pancreas, but also in other organs [31, 32]. However, although these modifications of pancreatic tissue structure have been qualitatively described and increased stromal collagen content has been reported in PDAC [33, 34], only a few studies have been focused to the quantitative assessment of the structure and the organization of stroma in this malignancy $[35,36]$.

It is now recognized that computer models are crucial for scientific procedures, and the modeling process represents a hypothetical-deductive approach in science [37, 38 . We performed this study with the aim to validate a novel method to quantify extra-cellular matrix deposition, geometrical spatial complexity and ECM degradation in histologic specimens from normal pancreatic tissue (nPA), inflammatory status (iPA) and PDAC. We used an automatically computer-aided image analysis system, which recognizes Sirius-red stained collagen fibers, quantifies the amount of pancreatic collagenic extracellular matrix (ECM) and its pattern, and simulates its degradation process. This novel methodology expands our knowledge of the ECM spatial organization and disposition in PDAC and may contribute knowledge relevant to clinically important aspects of this disease. 


\section{Methods}

\section{Patients}

Formalin-fixed and paraffin-embedded specimens were obtained from 7 patients with diagnosed PDAC (3 males and 4 females, age ranged from 62 to 78 years) and 6 subjects ( 3 males and 3 females, age ranged from 56 to 74 years) with chronic pancreatitis, who had previously undergone surgical resection for neoplasm or non-malignant diseases respectively (10 images for case at $20 \times$ objective). As controls, pancreatic tissue specimens with no evident pathology (again 10 images for case at $20 \times$ objective) were obtained by autopsy from 5 individuals (3 males and 2 females, age ranged from 36 to 76 years). Patients' clinical characteristics are reported in Table 1. Patients who underwent surgical procedure provided a written consent to the study participation.

\section{Histochemistry}

Two consecutive 2- $\mu$ m-thick sections were cut from each formalin-fixed, paraffin-embedded specimen. One was subsequently stained with haematoxylin \& eosin solution, and the other was stained with a freshly prepared PicroSirius red collagen staining solution [39].

\section{Computer-aided morphometric and fractal analysis}

For each Sirius-red stained section, ten "regions of interest" (ROIs) were digitized at $20 \times$ objective magnifications by using an Olympus microscope (Olympus, Italy). Ad hoc software automatically selects collagen fibers based on RGB color segmentation. The same image intensity level was used throughout the study. The surface area of the fibrosis, its distribution variability and fractal dimension were automatically calculated for each digitized ROI. In brief, we geometrically defined the following:

a. Sirius-red stained ECM as a set of irregularly shaped objects (collagen fragments or islets) that could be

\begin{tabular}{ll}
$\begin{array}{l}\text { Table } \mathbf{1} \text { Clinical } \\
\text { in the study }\end{array}$ & characteristics of \\
\hline Patients & 18 \\
Sex & Male: $9(50 \%)$ \\
& Female: $9(50 \%)$ \\
Age (years) & $65.8 \pm 2.55($ range $36-78)$ \\
Histological diagnosis & PDAC: 7 \\
& CP: 6 \\
Ca $19-9(\mathrm{U} / \mathrm{ml})$ & NP: 5 \\
CEA (ng/ml) & $61.3 \pm 24.4$ (range $2.5-182)$ \\
\hline
\end{tabular}

PDAC pancreatic ductal adenocarcinoma, CP chronic pancreatitis, NP normal pancreas, SE standard error distinguished from the remaining tissue by their chemical affinity to Sirius red dye (Fig. 1a-c).

b. Sirius-red stained ECM surface as the sum of all the areas of the collagen islets, expressed as a percentage of the pancreatic section surface area excluding any unfilled natural spaces and tissue-free spaces resulting from specimen manipulation.

c. 2-D ECM fractal surface dimension as a measure of its space-filling properties, which was automatically estimated by means of the box-counting method [40, 41] using the formula:

$D=\lim _{\varepsilon \rightarrow 0} \inf \frac{\log (N(\varepsilon))}{\log (1 / \varepsilon)}$

where $D$ is the box-counting fractal dimension, $\varepsilon$ the side length of the box, and $N(\varepsilon)$ the smallest number of boxes of side $\varepsilon$ required to cover the complete surface of interest of the object (i.e. containing useful information). As the zero limit cannot be applied to biological objects, the dimensions were calculated as $D=d$, where $\mathrm{d}$ is the slope of the graph of $\log [N(\varepsilon)]$ against $\log 1 / \varepsilon$. The $\log -\log$ graphs were plotted, the linear segments were identified using least squares regression, and their gradients were calculated using an iterative resistant line method, as previously described [42]. The concept of fractal dimension is shown in Fig. 2.

\section{Computer-aided ECM degradation simulation}

A model simulating the two-dimensional degradation of the Sirius-red stained irregularly shaped collagen fibers (i.e. collagen islets) was developed (Fig. 3a-c). The adopted mathematical operation is called "erosion" [43, 44]. Erosion (i.e. removes pixels on object boundaries) is one of two fundamental operations (the other being dilation, i.e. adds pixels to the boundaries of objects in an image) in morphological image processing from which all other morphological operations are based. This mathematical function is dependent on the shape of the object. In other words the process of erosion is dependent on the degree of irregularity of the object. For any temporal cycle, the function eliminates the isolated pixels from the background and erodes the boundaries of the collagen fibers. As the ECM consists of a set of irregularly shaped fibers [45-50] with different size, the dynamical process of erosion depends on the fibers shape, size and spatial pattern [51]. At any cycle, the model automatically evaluated the "Sirius-red stained ECM surface" as the sum of the areas of the collagen islets, expressed in percentage. The "number of cycles" is defined as the time necessary to obtain a Sirius-red stained ECM surface equal to $0 \%$. The higher is the irregularity of the collagen fibers, the 

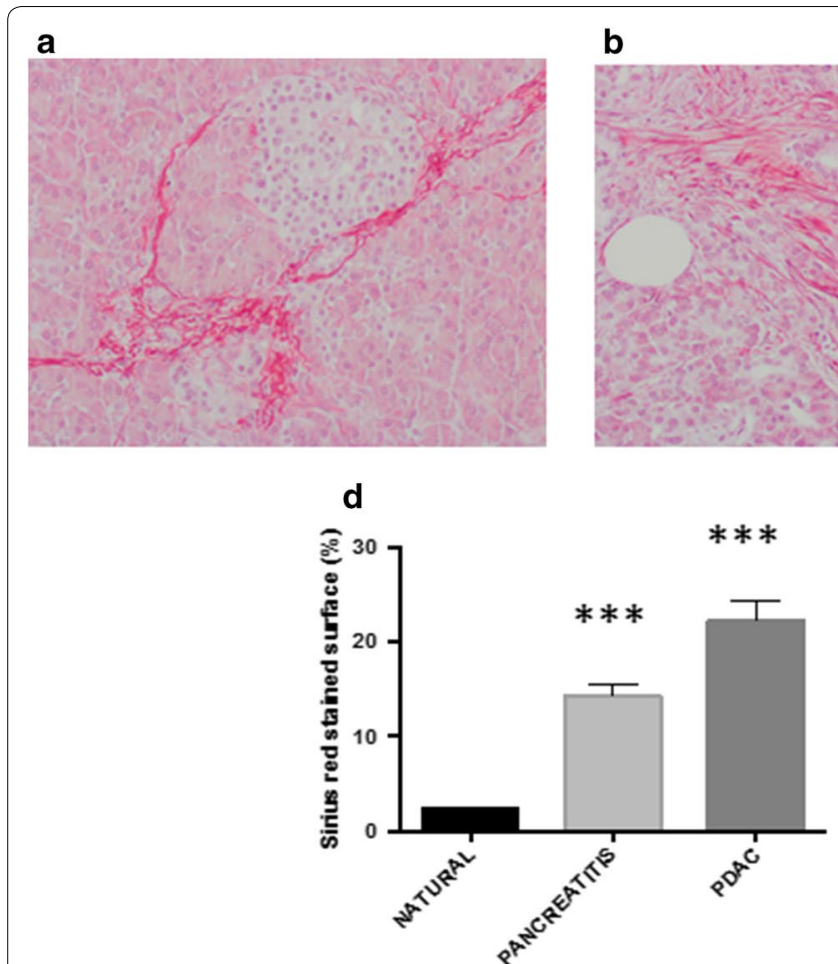

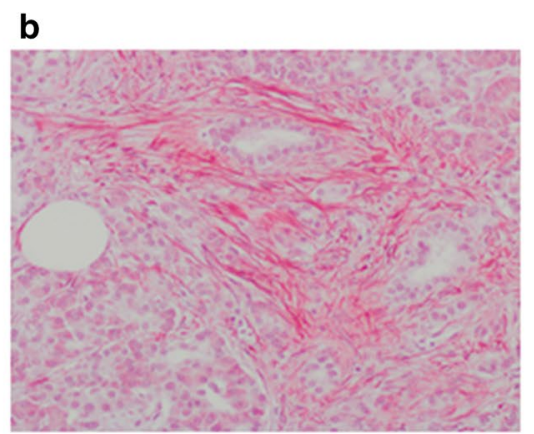

C

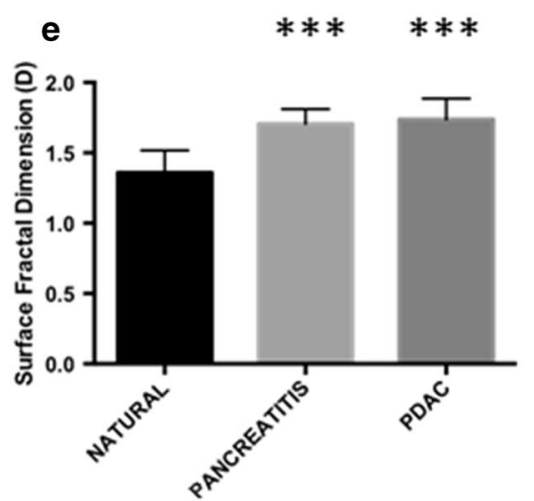

Fig. 1 The deposition of collagen extracellular-matrix drastically increases from natural pancreas (a) to pancreatitis (b) and adenocarcinoma (c). We found statistically significant difference when comparing the percentage of ECM matrix (d) and its 2-D surface fractal dimension (e). ${ }^{* * *} p<0.0001$ by Student's t-test

higher is the number of cycle necessary to complete the degradation process. Thus, such number of cycles is a marker of fibers shape irregularity.

In addition to the number of cycles necessary to completely erode the Sirius-red stained ECM, we also considered a second parameter, i.e., the "mean velocity of degradation" (i.e. the amount of ECM erased during each cycle) obtained dividing the initial ECM surface with the number of cycles.

\section{Statistical analysis}

Data are expressed as mean \pm standard deviation (unless otherwise specified), and were analyzed using Statistica software (StatSoft Inc., Tulsa, OK, USA) and GraphPad Prism software version 7.0 application (GraphPad Software, San Diego, CA, USA).

Data comparisons were performed by using the unpaired Student $\mathrm{t}$-test. $\mathrm{P}$ values of less than 0.05 were considered statistically significant.

\section{Results}

\section{Morphometric and fractal analysis}

In keeping with previous studies, we found a statistically significant progressive increase of collagen extra-cellular matrix deposition from normal pancreatic tissue (nPA) to inflammatory status (iPA) to pancreatic ductal adenocarcinoma PDAC [nPA: $2.23 \pm 0.28 \%$, iPA: $14.27 \pm 1.27 \%$, PDAC: $22.30 \pm 2.031 \%$; $<<0.0001$ ] (Fig. 1d). With regard to the 2-D fractal surface dimension (i.e. space-filling property of a set of irregularly shaped ECM fibers) we found a statistically significant difference $(p<0.0001)$ in the geometrical spatial complexity of ECM between $\mathrm{nPA}(1.35 \pm 0.02)$ versus iPA $(1.70 \pm 0.01)$ and PDAC $(1.73 \pm 0.01)$, but no differences were found between iPA and PDAC (Fig. 1e).

\section{Degradation simulation}

As shown in Fig. 3d, we found a statistically significant difference $(\mathrm{p}<0.001)$ when comparing the number of cycles necessary to erode the Sirius-red stained ECM in nPA (12.94 \pm 0.94$)$ versus iPA $(21.27 \pm 1.43)$ and PDAC $(27.61 \pm 1.43)$. Difference between iPA and PDAC was also significant $(\mathrm{p}<0.003)$. In addition, when we analyzed the mean velocity of Sirius-red stained ECM degradation (Fig. 3e) we found that this process is faster in iPA and PDAC than in nPA and that the difference was statistically significant $(\mathrm{p}<0.001)$. In contrast, no significant differences were found when comparing the simulated velocity of degradation between pancreatitis and PDAC 


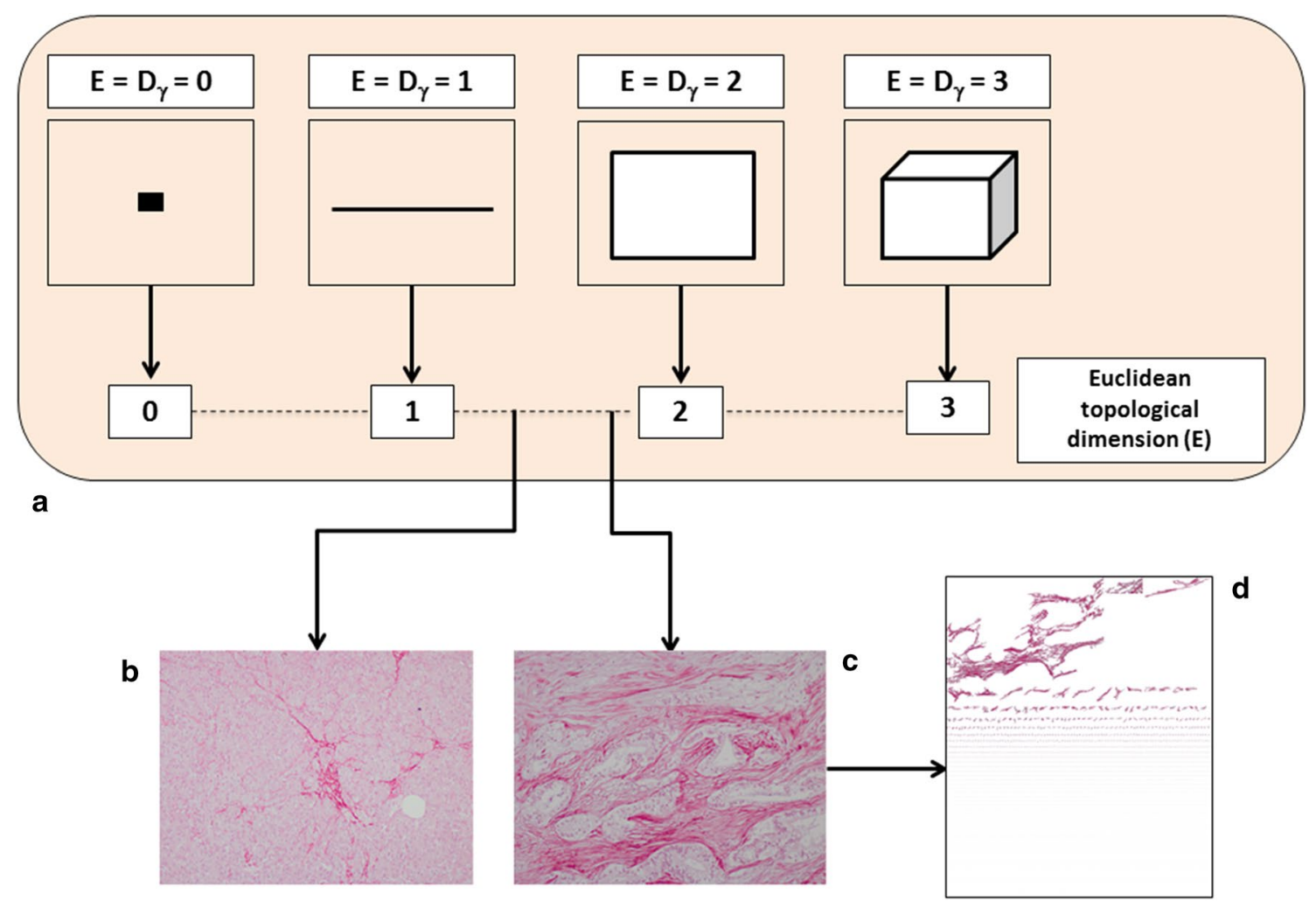

Fig. 2 The fractal (i.e. non-integer) dimension is a real number that can be attributed to every natural object. The topological dimension of an object is indicated with the symbol Dy, whereas the fractional dimension simply with D. For all Euclidean figures, Dy and D are coincident, i.e., $\mathrm{D} \gamma=\mathrm{D}(\mathbf{a})$. This equality is not valid for the natural, including biological, objects. Natural objects can be roughly represented by Euclidean shapes (i.e., a tree resembles a cylinder, the sun is similar to a sphere, a mountain can be interpreted as a cone), but in reality, these shapes are not Euclidean figures. As suggested by Benoit Mandelbrot, it is possible to determine the Hausdorff-Besicovitch dimension or FD, of irregularly shaped objects through the covering procedure of the topological space of the object being measured. The software automatically estimates the 2D-fractal dimension of Sirius red stained pancreatic ECM (b, c). The more D tends to 2 the more the analyzed conformation tends to fill a 2D space and the greater it's the geometrical complexity. Pancreatic desmoplasia consists of a set of irregularly shaped "collagen islets" (d)

(Fig. 3e). Table 2 reports all the data obtained by analyzing the geometrical features of Sirius-red stained ECM.

\section{Discussion}

Pancreatic cancer is characterized by the formation of a dense, "desmoplastic" stroma (Fig. 1) [52, 53]. Whether this stroma drives the progression of PDAC or acts as a defense [54-56], still remains controversial. It has been shown that high stromal activity, as assessed by $\alpha$-smooth muscle actin ( $\alpha$-SMA) expression, is associated with a poor prognosis in patients with PDAC [57]. Similarly, the high expression of ECM proteins such as SPARC [58] and periostin [59] is associated with a poor prognosis. Recently, Whatcott et al. observed a significant negative correlation between patient survival and ECM deposition in primary tumors [60].

Pancreatic fibrogenesis remains one of the most complex biological phenomena [61]. It is a dynamic process that is discontinuous in space and time, but advances through qualitatively different states. The non-linear progression of these states generates a complex structure that irregularly fills the surrounding environment (Fig. 2).

Several methods have been proposed to histologically quantify the stromal reaction in pancreatic carcinogenesis [62]. However, they have a number of substantial limitations, mainly due to the complex biology characterizing pancreatic ECM, and the highly irregular geometry that the stromal network assumes in real space, which cannot be quantified using the principles of Euclidean geometry (only capable of interpreting regular and smooth objects). The main feature of the newly generated ECM is the structural diversity of the Sirius-red collagen islets, shapes, sizes and distribution pattern [45-50]. Quantitative descriptors of ECM geometrical complexity can be usefully abstracted from the fractal geometry [45-50]. In general terms, fractal objects are mainly characterized by four properties: (a) the irregularity of their shape; (b) the self-similarity of their structure; (c) their non-integer 


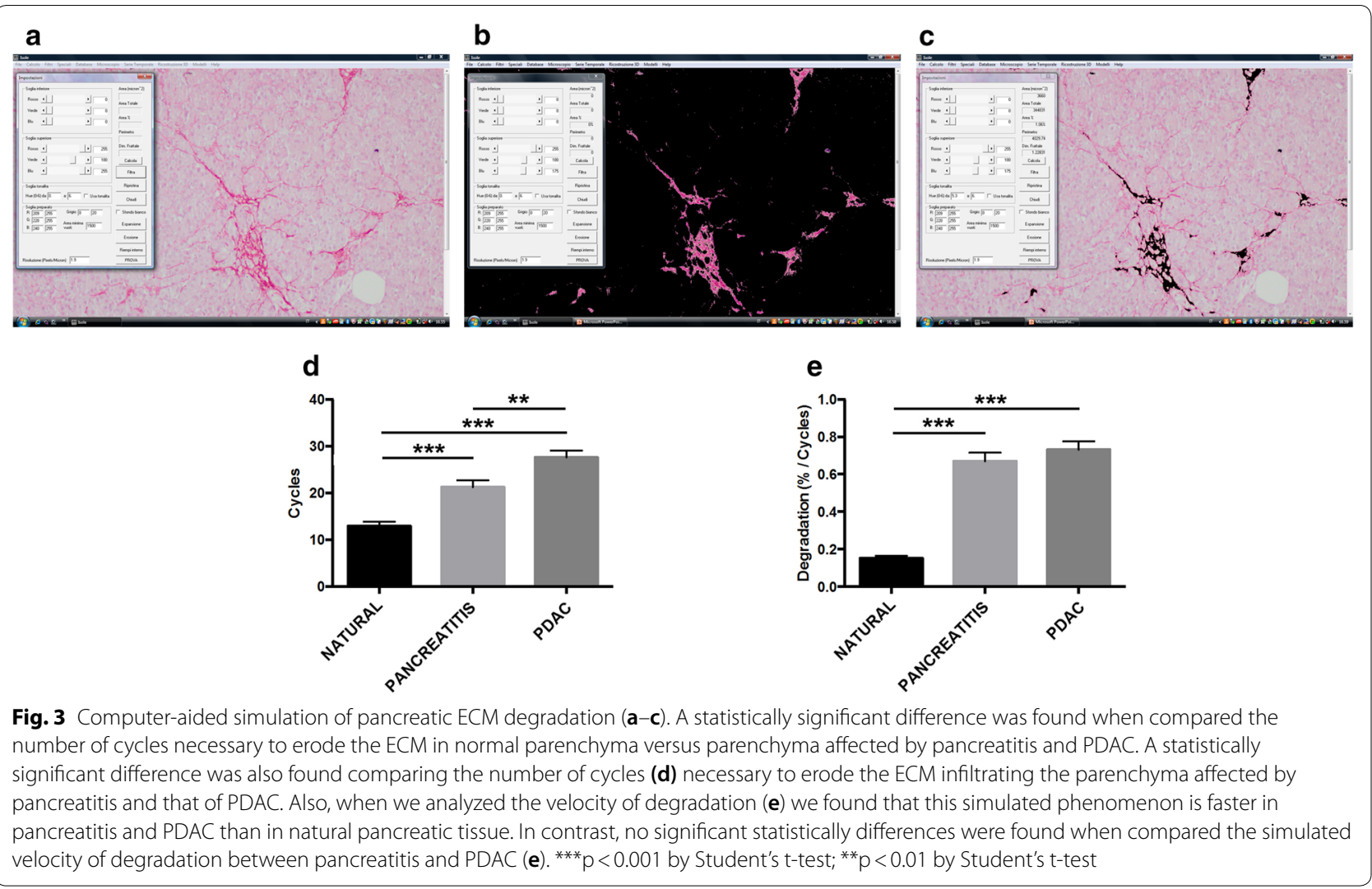

Table 2 Geometrical features of Sirius red stained pancreatic collagenic ECM and simulated degradation

\begin{tabular}{lllllllll}
\hline & \multicolumn{3}{l}{ Computer-aided } & morphometric analysis & & \multicolumn{2}{l}{ Computer modeling } \\
\cline { 2 - 3 } & Cases & ROI & $\begin{array}{l}\text { Sirius-red stained } \\
\text { surface (\%) }\end{array}$ & $\begin{array}{l}\text { Sirius-red stained collagenic ECM } \\
\text { geometrical complexity (D) }\end{array}$ & & Erosion cycles (n) & Erosion velocity \\
Natural pancreatic tissue & 5 & 50 & $2.23 \pm 0.28$ & $1.36 \pm 0.02$ & $12.94 \pm 0.94$ & $0.15 \pm 0.01$ \\
Pancreatitis & 6 & 60 & $14.27 \pm 1.27$ & $1.70 \pm 0.01$ & $21.27 \pm 1.43$ & $0.67 \pm 0.04$ \\
PDAC & 7 & 70 & $22.30 \pm 2.03$ & $1.74 \pm 0.01$ & & $27.61 \pm 1.43$ & $0.73 \pm 0.04$ \\
\hline
\end{tabular}

Data are expressed as mean \pm standard deviation

$R O /$ region of interest, $P D A C$ pancreatic adenocarcinoma

or fractal dimension; and (d) scaling, which means that the measured properties depend on the scale at which they are measured. An object is geometrically self-similar when every smaller piece of the object is an exact, or nearly exact, duplicate of the whole object. Statistical self-similarity concerns biological objects, including many anatomic forms [63].

Here, we have developed an innovative computer-aided methodology to investigate some geometrical features of the stroma in chronic pancreatitis and PDAC. To our knowledge, this is the first study where advanced mathematical modeling techniques, such as algorithms for fractal and degradation assessment, were used for the study of the pancreatic tissue.
Modeling is the process of generating mathematical models. A scientific model can provide a way to read elements easily, which have been broken down to a simpler form. A model is a simplified representation of a system at some particular point in time or space intended to promote understanding of the real system. A simulation is the manipulation of a model in such a way that it operates in time or space to compress it, thus enabling one to perceive the interactions that would not otherwise be apparent because of their separation in time or space. A simulation is the implementation of a model. In other words, simulation is the imitation of the operation of a real-world process or system over time while the model represents the system itself, whereas the simulation 
represents the operation of the system over time. In line with previous studies [33-36], our findings suggest a significant higher amount of collagen fibers deposition in inflammatory and neoplastic pancreatic tissue in comparison with natural pancreas. Furthermore, the fractal analysis disclosed that collagen fibers in inflammatory and neoplastic tissue show a more irregular surface as compared to those seen in normal tissue (Fig. 1e). These data support the hypothesis that during the inflammatory and neoplastic states involving the pancreas a significant modification of stromal components occurs either in terms of total amount and spatial organization. Although our studies provides no direct assessment of tissue stiffness in chronic pancreatitis and in PDAC, it is conceivable to suppose that the deposited ECM leads to increase in both tissue solid stress and tissue interstitial fluid pressures, both of which may mediate vascular compression and dysfunction [64-66]. These changes may also explain the scarce drug penetration observed in PDAC tissue [64].

In addition to the fractal analyses, to investigate the dynamics of collagenic ECM degradation we applied the morphological "erosion" function (Fig. 3a-c). Our model revealed for the first time a statistically significant difference when compared the number of cycles (i.e. time) necessary to erode the ECM: lower in nPA and higher in iPA and PDAC (Fig. 3d). This finding might reflect not only the increased amount of ECM observed in iPA and PDAC, but also the collagenic ECM conformation. Additionally, when we analyzed the velocity of degradation (i.e. the amount of ECM erased during each cycle) we found that this phenomenon is faster in iPA and PDAC while no differences were found between pancreatitis and PDAC (Fig. 3e). This finding means a presence of more compact, and thus difficult to erode, collagen in neoplastic and inflammatory conditions.

Of note, our methodology may have future applications of direct clinical relevance. Indeed, we hypothesize that fractal analyses may be applied in an in vivo context, for improved geometrical and morphological analyses of pancreatic images from radiological examinations. Such as approach appears plausible, as fractal analysis have been already applied for the study of radiological images [67], though this has never been attempted for the study of the pancreas.

\section{Conclusions}

The results generated by our automatic computer methodology suggest that three variables are important in the quantitative evaluation of pancreatic desmoplasia: (a) the size, (b) the shape, and (c) the pattern of arrangement of collagen fibers. In view of these preliminary results, it may be hypothesized that the complex modifications of the ECM conformation in addition to the changes of the ECM composition might promote an increase in pancreatic tissue stiffness. This condition is associated with an increased risk of cancer development. Despite the low number of enrolled subjects and the absence of direct assessment of the relationship between extra-cellular matrix deposition, ECM degradation simulation and pancreatic tissue stiffness, this study represents a pilot study for further well-designed and adequately-sized studies to confirm these preliminary but promising results. It is however indubitable that viewing pancreatic cancer as a system that is dynamically complex in time and space will probably reveal more about its underlying behavioral characteristics. This way of thinking may further help to clarify concepts, interpret new and old experimental data, indicate alternative experiments and categorize the acquired knowledge on the basis of the similitude and/or shared behaviors of very different tumors. It is encouraging that mathematics, theoretics, biology and medicine continue to contribute together towards a common quantitative understanding of cancer complexity.

\section{Abbreviations}

PDAC: pancreatic ductal adenocarcinoma; pcECM: pancreatic collagenic extra-cellular matrix; nPA: pancreatic natural; iPA: pancreatic inflammatory; ROI: regions of interest; SPARC: secreted protein acidic and rich in cysteine.

\section{Authors' contributions}

FG conceived the study, carried out the experimental studies, interpreted the results and wrote the first draft of the article. SF conceived the study, interpreted the results and revised the draft. CR developed the computer image analysis system. AF and DB contributed to the design of the study. AT analyzed the data and revised the draft of the article. MM, LM, AD and ZM commented on drafts of the manuscript. DQ, EA and RL carried out the experimental studies, participated in data interpretation. EJ, IM, LM, MCI, RSB and LDT supervised and critically reviewed the manuscript. All authors read and approved the final manuscript.

\section{Author details}

${ }^{1}$ Department of Immunology and Inflammation, Humanitas Clinical and Research Center-IRCCS, Rozzano, Milan, Italy. ${ }^{2}$ Humanitas University, Rozzano, Milan, Italy. ${ }^{3}$ Internal Medicine Unit, Maggiore Hospital, Bologna, Italy. ${ }^{4}$ Anatomic Pathology Service, Maggiore Hospital, Bologna, Italy. 5"Michele Rodriguez" Foundation-Institute for Quantitative Measures in Medicine, Milan, Italy. ${ }^{6}$ Department of Pharmacy and Biotechnology (FaBiT), University of Bologna, Bologna, Italy. ${ }^{7}$ Surgery Unit, Maggiore Hospital, Bologna, Italy. ${ }^{8}$ Ultrasound Center Internal Medicine A, Maggiore Hospital, Bologna, Italy. ${ }^{9}$ CNR Institute of Neuroscience, Padua, Italy. ${ }^{10}$ Department of Pathology, Humanitas Clinical and Research Center-IRCCS, Rozzano, Milano, Italy. ${ }^{11}$ Kiromic Biopharma, Inc., Houston, TX, USA. ${ }^{12}$ Department of Gastroenterology, Hepatology \& Nutrition, Division of Internal Medicine, The University of Texas MD Anderson Cancer, Houston, TX, USA. ${ }^{13}$ Histology Core, Humanitas Clinical and Research Center-IRCCS, Rozzano, Milan, Italy.

\section{Acknowledgements}

The authors are grateful to Teri Fields for her manuscript editing and Simonetta Righi for her precious effort in the recruitment of literature references.

\section{Competing interests}

The authors declare that they have no competing interests. 


\section{Availability of data and materials}

All data generated or analyzed during this study are included in this published article.

\section{Consent for publication}

Not applicable.

\section{Ethics approval and consent to participate}

Patients who underwent surgical procedure provided a written consent to the study participation.

\section{Funding}

None.

\section{Publisher's Note}

Springer Nature remains neutral with regard to jurisdictional claims in published maps and institutional affiliations.

Received: 2 October 2018 Accepted: 21 February 2019

Published online: 28 February 2019

\section{References}

1. Saif MW. Advancements in the management of pancreatic cancer: 2013 JOP. 2013;14(2):112-8

2. Siegel RL, Miller KD, Jemal A. Cancer statistics, 2015. Cancer J Clin. 2015;65(1):5-29.

3. Wolfgang $C L$, Herman JM, Laheru DA, Klein AP, Erdek MA, Fishman EK, Hruban RH. Recent progress in pancreatic cancer. Cancer J Clin. 2013;63(5):318-48.

4. Cowan RW, Maitra A. Genetic progression of pancreatic cancer. Cancer J. 2014;20(1):80-4

5. Kozak G, Blanco FF, Brody JR. Novel targets in pancreatic cancer research. Semin Oncol. 2015:42(1):177-87.

6. Murphy SJ, Hart SN, Lima JF, Kipp BR, Klebig M, Winters JL, Szabo C, Zhang L, Eckloff BW, Petersen GM, et al. Genetic alterations associated with progression from pancreatic intraepithelial neoplasia to invasive pancreatic tumor. Gastroenterology. 2013;145(5):1098-109.

7. Thomas JK, Kim MS, Balakrishnan L, Nanjappa V, Raju R, Marimuthu A, Radhakrishnan A, Muthusamy B, Khan AA, Sakamuri S, et al. Pancreatic cancer database: an integrative resource for pancreatic cancer. Cancer Biol Ther. 2014;15(8):963-7.

8. Jones S, Zhang X, Parsons DW, Lin JC, Leary RJ, Angenendt P, Mankoo $\mathrm{P}$, Carter $\mathrm{H}$, Kamiyama $\mathrm{H}$, Jimeno A, et al. Core signaling pathways in human pancreatic cancers revealed by global genomic analyses. Science. 2008;321(5897):1801-6.

9. Matsuda Y, Ishiwata T, Izumiyama-Shimomura N, Hamayasu H, Fujiwara M, Tomita K, Hiraishi N, Nakamura K, Ishikawa N, Aida J, et al. Gradual telomere shortening and increasing chromosomal instability among PanIN grades and normal ductal epithelia with and without cancer in the pancreas. PLoS ONE. 2015;10(2):e0117575.

10. Kanda M, Matthaei H, Wu J, Hong SM, Yu J, Borges M, Hruban RH, Maitra A, Kinzler K, Vogelstein B, et al. Presence of somatic mutations in most early-stage pancreatic intraepithelial neoplasia. Gastroenterology. 2012;142(4):730-3.

11. Bardeesy N, DePinho RA. Pancreatic cancer biology and genetics. Nat Rev Cancer. 2002;2(12):897-909.

12. Maitra A, Hruban RH. Pancreatic cancer. Ann Rev Pathol. 2008;3:157-88.

13. Tanase CP, Neagu Al, Necula LG, Mambet C, Enciu AM, Calenic B, Cruceru $\mathrm{ML}$, Albulescu R. Cancer stem cells: involvement in pancreatic cancer pathogenesis and perspectives on cancer therapeutics. World J Gastroenterol. 2014;20(31):10790-801.

14. Bissell MJ, Kenny PA, Radisky DC. Microenvironmental regulators of tissue structure and function also regulate tumor induction and progression: the role of extracellular matrix and its degrading enzymes. Cold Spring Harb Symp Quant Biol. 2005;70:343-56.

15. Chu X, Jin Q, Chen H, Wood GC, Petrick A, Strodel W, Gabrielsen J, Benotti P, Mirshahi T, Carey DJ, et al. CCL20 is up-regulated in non-alcoholic fatty liver disease fibrosis and is produced by hepatic stellate cells in response to fatty acid loading. J Transl Med. 2018;16(1):108.

16. Sherman MH. Stellate Cells in Tissue Repair, Inflammation, and Cancer Annu Rev Cell Dev Biol. 2018;34:333-55.

17. Rucki AA, Zheng L. Pancreatic cancer stroma: understanding biology leads to new therapeutic strategies. WJG. 2014;20(9):2237-46.

18. Hermano E, Meirovitz A, Meir K, Nussbaum G, Appelbaum L, Peretz T, Elkin M. Macrophage polarization in pancreatic carcinoma: role of heparanase enzyme. J Natl Cancer Inst. 2014;106:12.

19. Sideras K, Braat H, Kwekkeboom J, van Eijck CH, Peppelenbosch MP, Sleijfer S, Bruno M. Role of the immune system in pancreatic cancer progression and immune modulating treatment strategies. Cancer Treat Rev. 2014;40(4):513-22.

20. Lunardi S, Muschel RJ, Brunner TB. The stromal compartments in pancreatic cancer: are there any therapeutic targets? Cancer Lett. 2014;343(2):147-55

21. Li CX, Cui LH, Zhuo YZ, Hu JG, Cui NQ, Zhang SK. Inhibiting autophagy promotes collagen degradation by regulating matrix metalloproteinases in pancreatic stellate cells. Life Sci. 2018;208:276-83.

22. Neesse A, Algul H, Tuveson DA, Gress TM. Stromal biology and therapy in pancreatic cancer: a changing paradigm. Gut. 2015;64(9):1476-84.

23. Neesse A, Michl P, Frese KK, Feig C, Cook N, Jacobetz MA, Lolkema MP, Buchholz M, Olive KP, Gress TM, et al. Stromal biology and therapy in pancreatic cancer. Gut. 2011;60(6):861-8.

24. Laklai H, Miroshnikova YA, Pickup MW, Collisson EA, Kim GE, Barrett AS, Hill RC, Lakins JN, Schlaepfer DD, Mouw JK, et al. Genotype tunes pancreatic ductal adenocarcinoma tissue tension to induce matricellular fibrosis and tumor progression. Nat Med. 2016;22(5):497-505.

25. Nieskoski MD, Marra K, Gunn JR, Hoopes PJ, Doyley MM, Hasan T, Trembly BS, Pogue BW. Collagen complexity spatially defines microregions of total tissue pressure in pancreatic cancer. Sci Rep. 2017:7(1):10093.

26. Jiang $\mathrm{H}$, Hegde $\mathrm{S}$, DeNardo DG. Tumor-associated fibrosis as a regulator of tumor immunity and response to immunotherapy. CII. 2017;66(8):1037-48

27. Waghray M, Yalamanchili M, di Magliano MP, Simeone DM. Deciphering the role of stroma in pancreatic cancer. Curr Opin Gastroenterol. 2013;29(5):537-43.

28. Bissell MJ, Radisky D. Putting tumours in context. Nat Rev Cancer. 2001;1(1):46-54.

29. Miles FL, Sikes RA. Insidious changes in stromal matrix fuel cancer progression. MCR. 2014;12(3):297-312.

30. Ingber DE. Tensegrity-based mechanosensing from macro to micro. Prog Biophys Mol Biol. 2008;97(2-3):163-79.

31. Handorf AM, Zhou Y, Halanski MA, Li WJ. Tissue stiffness dictates development, homeostasis, and disease progression. Organogenesis. 2015;11(1):1-15.

32. Paszek MJ, Zahir N, Johnson KR, Lakins JN, Rozenberg Gl, Gefen A, Reinhart-King CA, Margulies SS, Dembo M, Boettiger D, et al. Tensional homeostasis and the malignant phenotype. Cancer Cell. 2005;8(3):241-54.

33. Nielsen MF, Mortensen MB, Detlefsen S. Key players in pancreatic cancerstroma interaction: cancer-associated fibroblasts, endothelial and inflammatory cells. WJG. 2016;22(9):2678-700.

34. Zhan HX, Zhou B, Cheng YG, Xu JW, Wang L, Zhang GY, Hu SY. Crosstalk between stromal cells and cancer cells in pancreatic cancer: new insights into stromal biology. Cancer Lett. 2017;392:83-93.

35. Drifka CR, Tod J, Loeffler AG, Liu Y, Thomas GJ, Eliceiri KW, Kao WJ. Periductal stromal collagen topology of pancreatic ductal adenocarcinoma differs from that of normal and chronic pancreatitis. Mod Pathol. 2015;28(11):1470-80.

36. Bever KM, Sugar EA, Bigelow E, Sharma R, Laheru D, Wolfgang $C L$, Jaffee EM, Anders RA, De Jesus-Acosta A, Zheng L. The prognostic value of stroma in pancreatic cancer in patients receiving adjuvant therapy. HPB. 2015;17(4):292-8

37. Massoud TF, Hademenos GJ, Young WL, Gao E, Pile-Spellman J, Vinuela F Principles and philosophy of modeling in biomedical research. FASEB J. 1998;12(3):275-85.

38. Weiss JN, Qu Z, Garfinkel A. Understanding biological complexity: lessons from the past. FASEB J. 2003;17(1):1-6.

39. Junqueira LC, Bignolas G, Brentani RR. Picrosirius staining plus polarization microscopy, a specific method for collagen detection in tissue sections. Histochem J. 1979:11(4):447-55. 
40. Mainieri R. On the equality of Hausdorff and box counting dimensions. Chaos. 1993;3(2):119-25.

41. Dai M, Liu X. The Hausdorff and box-counting dimensions of a class of recurrent sets. Chaos Solitons Fractals. 2008;36(3):532-8.

42. Grizzi F, Russo C, Colombo P, Franceschini B, Frezza EE, Cobos E, ChirivaInternati M. Quantitative evaluation and modeling of two-dimensional neovascular network complexity: the surface fractal dimension. BMC Cancer. 2005;5:14.

43. Serra JP. Image analysis and mathematical morphology, vol. 1. Cambridge: Academic Press; 1982.

44. Nguyen DM, Wagenhauser MU, Mehrkens D, Adam M, Tsao PS, Ramasubramanian AK. An automated algorithm to quantify collagen distribution in aortic wall. J Histochem Cytochem. 2018. https://doi. org/10.1369/0022155418814231.

45. Dioguardi N, Franceschini B, Aletti G, Russo C, Grizzi F. Fractal dimension rectified meter for quantification of liver fibrosis and other irregular microscopic objects. Anal Quant Cytol Histol. 2003;25(6):312-20.

46. Soda G, Nardoni S, Bosco D, Grizzi F, Dioguardi N, Melis M. Fractal analysis of liver fibrosis. Pathologica. 2003;95(2):98-102.

47. Dioguardi N, Grizzi F, Bossi P, Roncalli M. Fractal and spectral dimension analysis of liver fibrosis in needle biopsy specimens. Anal Quant Cytol Histol. 1999;21(3):262-6.

48. Dioguardi N, Grizzi F, Fiamengo B, Russo C. Metrically measuring liver biopsy: a chronic hepatitis $B$ and $C$ computer-aided morphologic description. World J Gastroenterol. 2008;14(48):7335-44.

49. Grizzi F, Russo C, Franceschini B, Di Rocco M, Torri V, Morenghi E, Fassati $L R$, Dioguardi N. Sampling variability of computer-aided fractal-corrected measures of liver fibrosis in needle biopsy specimens. World J Gastroenterol. 2006;12(47):7660-5.

50. Dioguardi N, Grizzi F, Franceschini B, Bossi P, Russo C. Liver fibrosis and tissue architectural change measurement using fractal-rectified metrics and Hurst's exponent. World J Gastroenterol. 2006;12(14):2187-94.

51. Chen Q. Computer simulation of solid particle erosion. Wear. 2003;254(2-3):203-10.

52. Brower V. Genomic research advances pancreatic cancer's early detection and treatment. J Natl Cancer Inst. 2015;107(7):djv195.

53. Hidalgo M. Pancreatic cancer. N Engl J Med. 2010;362(17):1605-17.

54. Gore J, Korc M. Pancreatic cancer stroma: friend or foe? Cancer Cell. 2014;25(6):711-2.

55. Leake I. Pancreatic cancer: surprising role for fibrosis. Nat Rev Gastroenterol Hepatol. 2014;11(7):396.

56. Ozdemir BC, Pentcheva-Hoang T, Carstens JL, Zheng X, Wu CC, Simpson TR, Laklai H, Sugimoto H, Kahlert C, Novitskiy SV, et al. Depletion of carcinoma-associated fibroblasts and fibrosis induces immunosuppression and accelerates pancreas cancer with reduced survival. Cancer Cell. 2014;25(6):719-34.

57. Erkan M, Michalski CW, Rieder S, Reiser-Erkan C, Abiatari I, Kolb A, Giese NA, Esposito I, Friess H, Kleeff J. The activated stroma index is a novel and independent prognostic marker in pancreatic ductal adenocarcinoma. Clin Gastroenterol Hepatol. 2008;6(10):1155-61.

58. Mantoni TS, Schendel RR, Rodel F, Niedobitek G, Al-Assar O, Masamune A, Brunner TB. Stromal SPARC expression and patient survival after chemoradiation for non-resectable pancreatic adenocarcinoma. Cancer Biol Ther. 2008;7(11):1806-15

59. Erkan M, Kleeff J, Gorbachevski A, Reiser C, Mitkus T, Esposito I, Giese T, Buchler MW, Giese NA, Friess H. Periostin creates a tumor-supportive microenvironment in the pancreas by sustaining fibrogenic stellate cell activity. Gastroenterology. 2007;132(4):1447-64.

60. Whatcott CJ, Diep CH, Jiang P, Watanabe A, LoBello J, Sima C, Hostetter G, Shepard HM, Von Hoff DD, Han H. Desmoplasia in primary tumors and metastatic lesions of pancreatic cancer. Clin Cancer Res. 2015;21(15):3561-8

61. Masamune A, Shimosegawa T. Pancreatic stellate cells: a dynamic player of the intercellular communication in pancreatic cancer. Clin Res Hepatol Gastroenterol. 2015;39(Suppl 1):S98-103.

62. Kong X, Li L, Li Z, Xie K. Targeted destruction of the orchestration of the pancreatic stroma and tumor cells in pancreatic cancer cases: molecular basis for therapeutic implications. Cytokine Growth Factor Rev. 2012;23(6):343-56.

63. Di leva A, Grizzi F, Jelinek H, Pellionisz AJ, Losa GA. Fractals in the neurosciences, part l: general principles and basic neurosciences. Neurosci. 2013;20(4):403-17.

64. Provenzano PP, Cuevas C, Chang AE, Goel VK, Von Hoff DD, Hingorani SR. Enzymatic targeting of the stroma ablates physical barriers to treatment of pancreatic ductal adenocarcinoma. Cancer Cell. 2012;21(3):418-29.

65. Stylianopoulos T, Martin JD, Chauhan VP, Jain SR, Diop-Frimpong B, Bardeesy N, Smith BL, Ferrone CR, Hornicek FJ, Boucher Y, et al. Causes, consequences, and remedies for growth-induced solid stress in murine and human tumors. Proc Natl Acad Sci USA. 2012;109(38):15101-8.

66. Fiorino S, Bacchi-Reggiani L, Pontoriero L, Gallo C, Chili E, Masetti M, Zanini N, Grondona A, Silvestri T, Deleonardi G, et al. Tensegrity model hypothesis: may this paradigm be useful to explain hepatic and pancreatic carcinogenesis in patients with persistent hepatitis B or hepatitis $C$ virus infection? JOP. 2014;15(2):151-64.

67. Czyz M, Kapinas A, Holton J, Pyzik R, Boszczyk BM, Quraishi NA. The computed tomography-based fractal analysis of trabecular bone structure may help in detecting decreased quality of bone before urgent spinal procedures. Spine J. 2017:17(8):1156-62.
Ready to submit your research? Choose BMC and benefit from:

- fast, convenient online submission

- thorough peer review by experienced researchers in your field

- rapid publication on acceptance

- support for research data, including large and complex data types

- gold Open Access which fosters wider collaboration and increased citations

- maximum visibility for your research: over 100M website views per year

At $\mathrm{BMC}$, research is always in progress.

Learn more biomedcentral.com/submissions 